\title{
Uncertainty Relations for Joint Measurements of Noncommuting Observables
}

\author{
Masanao Ozawd \\ Graduate School of Information Sciences, Tôhoku University, Aoba-ku, Sendai, 980-8579, Japan
}

\begin{abstract}
Universally valid uncertainty relations are proven in a model independent formulation for inherent and unavoidable extra noises in arbitrary joint measurements on single systems, from which Heisenber's original uncertainty relation is proven valid for any joint measurements with statistically independent noises.
\end{abstract}

PACS numbers: 03.65.Ta, 03.67.-a

\section{INTRODUCTION}

Heisenberg's uncertainty principle [1, 2] represents a fundamental limit on measurements in quantum mechanics as a consequence of noncommutativity of canonical observables. However, its interpretation and formalization have been an issue of debate for many years.

Heisenberg [1] originally asserted the relation (Heisenberg's relation) that the product of the imprecisions of measurements of conjugate observables carried out simultaneously on a system has a lower bound determined by Planck's constant, as exemplified by the $\gamma$ ray microscope thought experiment. Some of previous investigations, on the one hand, partially supported the validity of Heisenberg's relation [3, 4, 5, 6, 7], and explained the consistency of Heisenberg's relation with the sensitivity limit of experimentally available optical detectors and amplifiers $[4,5,8,8,9 \mid$. On the other hand, there have been criticisms on the validity of Heisenberg's relation and its consequences 10, 11, 12, 13]. Moreover, recent investigations [14, 15, 16] have revealed that Heisenberg's relation is violated in a rather common class of measurements.

We have also different formalizations of the uncertainty principle introduced by Kennard [17] and Robertson [18]. Robertson's relation for a pair of observables $A$ and $B$ is formalized by the relation

$$
\Delta A \Delta B \geq \frac{1}{2}|\langle[A, B]\rangle|,
$$

where $\langle\cdots\rangle$ stands for the mean value in the given state, $\Delta A$ and $\Delta B$ are the standard deviations of $A$ and $B$, defined by $\Delta X=\left(\left\langle X^{2}\right\rangle-\langle X\rangle^{2}\right)^{1 / 2}$ for $X=A, B$, and the square bracket stands for the commutator, i.e., $[A, B]=A B-B A$. Equation (1) can be proven by a simple application of the Schwarz inequality. For two conjugate observables $Q$ and $P$, which satisfy the canonical commutation relation

$$
[Q, P]=i \hbar
$$

we obtain Kennard's relation 17]

$$
\Delta Q \Delta P \geq \frac{\hbar}{2}
$$

Robertson's and Kennard's relations are naturally interpreted as the limitation of state preparations or the limitation of the ideal independent measurements on identically prepared systems [19, 20]. Moreover, the standard deviation, a notion dependent on the state of the system but independent of the apparatus, cannot be identified with the imprecision of the apparatus such as the resolution power of the $\gamma$ ray microscope. Thus, it is still missing to correctly describe the unavoidable imprecisions inherent to joint measurements of noncommuting observables.

The purpose of this Letter is to present a correct, general, and relevant formalization of the uncertainty principle for joint measurements in the model independent formulation based on the notion of positive operator valued measures, now broadly accepted as the most general description of statistics of measurement outcomes 20, 21, 22, 23, 24, 25], and to show that measurements obeying Heisenberg's relation are generally characterized by the statistical independence of the noise in measurement.

\footnotetext{
* The results in this Letter were presented at A Satellite Workshop to EQIS'03: Non-locality of Quantum Mechanics and Statistical Inference, held at Kyoto Sangyo University, Kyoto, Japan, on September 8-9, 2003.

${ }^{\dagger}$ Electronic address: ozawa@mailaps.org
} 


\section{VIOLATION OF HEISENBERG'S RELATION}

Before discussing the general formulation, we shall see how Heisenberg's relation is commonly violated. The wellknown device to realize approximate simultaneous measurements of conjugate observables [3, 4, 9] is based on the fact that two independent pairs of conjugate observables $(Q, P)$ and $\left(Q^{\prime}, P^{\prime}\right)$ satisfy the commutation relation

$$
\left[Q-Q^{\prime}, P+P^{\prime}\right]=0
$$

where the independence ensures the relation $\left[Q, P^{\prime}\right]=\left[Q^{\prime}, P\right]=0$. Thus, we have an apparatus to simultaneously measure $Q_{-}=Q-Q^{\prime}$ and $P_{+}=P+P^{\prime}$ with arbitrary precision. The precise simultaneous measurement of $Q_{-}$ and $P_{+}$can be interpreted as an approximate simultaneous measurement of $Q$ and $P$, if we take the outputs from the above measurement to be the measured values of $Q$ and $P$. How precisely $Q$ has been measured is evaluated by the difference between the observable $Q$ to be measured and the observable $Q_{-}$actually measured. Thus, the root-mean-square (rms) noise $\epsilon(Q)=\left\langle\left(Q_{-}-Q\right)^{2}\right\rangle^{1 / 2}$ is a reasonable measure of imprecision for the $Q$ measurement. Analogously, so is the rms noise $\epsilon(P)=\left\langle\left(P_{+}-P\right)^{2}\right\rangle^{1 / 2}$ for the $P$ measurement. By $Q_{-}-Q=Q^{\prime}, P_{+}-P=P^{\prime}$, we have

$$
\begin{aligned}
& \epsilon(Q)=\left\langle Q^{\prime 2}\right\rangle^{1 / 2} \geq \Delta Q^{\prime}, \\
& \epsilon(P)=\left\langle P^{\prime 2}\right\rangle^{1 / 2} \geq \Delta P^{\prime},
\end{aligned}
$$

so that the relation (3), applied to the pair $\left(Q^{\prime}, P^{\prime}\right)$, concludes Heisenberg's relation

$$
\epsilon(Q) \epsilon(P) \geq \frac{\hbar}{2}
$$

Thus, the simultaneous measurement of commuting observables $Q_{-}$and $P_{+}$can be regarded as an approximate simultaneous measurement of conjugate observables $Q$ and $P$ which satisfies Heisenberg's relation.

Now, we consider other independent pairs $\left(Q, P_{+}\right)$and $\left(Q_{-},-P\right)$ of conjugate observables produced by the canonical transformation $Q \rightarrow Q, P \rightarrow P_{+}, Q^{\prime} \rightarrow Q_{-}$, and $P^{\prime} \rightarrow-P$. Then, the precise simultaneous measurement of $Q_{-}$ and $P_{+}$is also interpreted as an approximate simultaneous measurement of conjugate observables $Q$ and $P_{+}$. In this case, we obviously have $\epsilon\left(P_{+}\right)=0$. Thus, if Heisenberg's relation were to hold, we would have $\epsilon(Q)=\infty$. However, if $Q^{\prime}$ would be prepared to be $Q^{\prime}=0$, the precise $Q_{-}$measurement could be regarded as the precise $Q$ measurement. Indeed, if the mean position of $Q^{\prime}$ is prepared at the origin, i.e., $\left\langle Q^{\prime}\right\rangle=0$, then from Eq. (5) we have $\epsilon(Q)=\Delta Q^{\prime}$, so that we can make $\epsilon(Q)$ arbitrarily small, and we conclude

$$
\epsilon(Q) \epsilon\left(P_{+}\right)=0 \text {. }
$$

Thus, Heisenberg's relation is violated, and more significantly we have shown that the position $Q$ can be measured with arbitrary precision without disturbing the total momentum $P+P^{\prime}$.

Therefore, we have shown that the precise simultaneous measurement of commuting observables $Q_{-}$and $P_{+}$can be regarded, on the one hand, as an approximate simultaneous measurement of conjugate observables $Q$ and $P$ satisfying Heisenberg's relation and, on the other hand, as an effectively precise simultaneous measurement of conjugate observables $Q$ and $P_{+}$. In particular, both Eq. (7) and Eq. (8) hold mathematically for every state with $0<$ $\Delta Q, \Delta P, \Delta Q^{\prime}, \Delta P^{\prime}<0$.

This contradicts also a naive interpretation of the Wigner-Araki-Yanase theorem stating that only observables commuting with all the additively conserved quantities can be measured precisely [27, 28, 29, 30, 31, 32]. Our generalized uncertainty relations will clarify, among others, what limitation is generally posed in the above situation.

\section{JOINT PROBABILITY OPERATOR VALUED MEASURES}

Let us consider a quantum system $\mathbf{S}$ described by a Hilbert space $\mathcal{H}$ with two observables $A$ and $B$. We associate any joint measurement of observables $A$ and $B$ to an apparatus $\mathbf{A}(\mathbf{x}, \mathbf{y})$ with two output variables $\mathbf{x}$ and $\mathbf{y}$, where x measures $A$ and $\mathbf{y}$ measures $B$, even approximately. We assume that any joint measurements are carried out on a single systems to obtain, simultaneously or successively in time, the measured values of $A$ and $B$ in a common state just before the measurement. For simplicity, we assume that $\mathcal{H}$ is finite dimensional and that $\mathbf{x}$ and $\mathbf{y}$ take only finite number of values. It is now fairly well-known [20, 21, 22, 23, 24, 25] that to such an apparatus $\mathbf{A}$ we can associate a family $\{\Pi(x, y) \mid x, y \in \mathbf{R}\}$ of positive operators on $\mathcal{H}$ satisfying (i) $0 \leq \Pi(x, y) \leq I$, (ii) $\sum_{x, y} \Pi(x, y)=I$. Then, the joint probability of obtaining the outcomes $\mathbf{x}=x$ and $\mathbf{y}=y$ in the state $\psi$ is described by

$$
\operatorname{Pr}\{\mathbf{x}=x, \mathbf{y}=y \| \psi\}=\langle\psi|\Pi(x, y)| \psi\rangle .
$$


We shall call the above family $\{\Pi(x, y) \mid x, y \in \mathbf{R}\}$ the joint probability operator valued measure (POVM) of the apparatus $\mathbf{A}(\mathbf{x}, \mathbf{y})$. The joint POVM $\Pi(x, y)$ defines two marginal POVMs $\Pi^{A}(x)$ and $\Pi^{B}(y)$ by $\Pi^{A}(x)=\sum_{y} \Pi(x, y)$ and $\Pi^{B}(y)=\sum_{x} \Pi(x, y)$. The marginal POVMs $\Pi^{A}(x)$ and $\Pi^{B}(y)$ describe the output probability distributions for the $A$ measurement and the $B$ measurement, respectively, i.e., $\operatorname{Pr}\{\mathbf{x}=x \| \psi\}=\left\langle\psi\left|\Pi^{A}(x)\right| \psi\right\rangle$ and $\operatorname{Pr}\{\mathbf{y}=y \| \psi\}=$ $\left\langle\psi\left|\Pi^{B}(y)\right| \psi\right\rangle$.

From the Born rule for the probability distributions of observables, the joint $\operatorname{POVM~} \Pi(x, y)$ gives a precise $A$ measurement if and only if $\Pi^{A}(x)=E^{A}(x)$ for all $x \in \mathbf{R}$, where $E^{A}(x)$ is the projection on the subspace $\{\psi \in$ $\mathcal{H} \mid A \psi=x \psi\}$. Analogously, $\Pi(x, y)$ gives a precise $B$ measurement if and only if $\Pi^{B}(y)=E^{B}(y)$ for all $y \in \mathbf{R}$. It is well-known that $\Pi(x, y)$ gives both a precise $A$ measurement and a precise $B$ measurement if and only if

$$
\Pi(x, y)=E^{A}(x) E^{B}(y)
$$

for all $x, y \in \mathbf{R}\left[22\right.$. Since $\Pi(x, y)^{\dagger}=\Pi(x, y)$, we have $\left[E^{A}(x), E^{B}(y)\right]=0$ for all $x, y \in \mathbf{R}$, so that $[A, B]=0$. Thus, the precise simultaneous measurement of $A$ and $B$ is possible if and only if $A$ and $B$ commute. However, the above result does not exclude the possibility that there is a subspace $\mathcal{M}$ such that an effectively precise simultaneous measurement of $A$ and $B$ are possible for any state in $\mathcal{M}$ on which $[A, B] \neq 0$. For such problem the quantitative investigation is inevitable.

\section{MEASURING PROCESSES}

The above statistical description of measurement by the POVM is known to be consistent with a description of measuring process [24]. A measuring process for the joint $\operatorname{POVM~} \Pi(x, y)$ is defined to be a 5 -tuple $\left(\mathcal{K}, \xi, U, M_{1}, M_{2}\right)$ consisting of a Hilbert space $\mathcal{K}$, a state vector $\xi$ on $\mathcal{K}$, a unitary operator $U$ on $\mathcal{H} \otimes \mathcal{K}$, and commuting observables $M_{1}$ and $M_{2}$ on $\mathcal{K}$ such that

$$
\Pi(x, y)=\left\langle\xi\left|U^{\dagger}\left[I \otimes E^{M_{1}}(x) E^{M_{2}}(y)\right] U\right| \xi\right\rangle
$$

for any $x, y \in \mathbf{R}$, where $\langle\xi|\cdots| \xi\rangle$ is the partial mean on $\mathcal{K}$, i.e., $\langle\psi|\langle\xi|\cdots| \xi\rangle| \psi\rangle=\langle\psi \otimes \xi|\cdots| \psi \otimes \xi\rangle$ for all $\psi \in \mathcal{H}$. If the joint measurement described by $\Pi(x, y)$ is carried out by a measuring process $\left(\mathcal{K}, \sigma, U, M_{1}, M_{2}\right)$, the input state $\rho$ is changed to the conditional output state $\rho_{\{\mathbf{x}=x, \mathbf{y}=y\}}$

$$
\rho_{\{\mathbf{x}=x, \mathbf{y}=y\}}=\frac{\operatorname{Tr}_{\mathcal{K}}\left\{U(\rho \otimes|\xi\rangle\langle\xi|) U^{\dagger}\left[I \otimes E^{M_{1}}(x) E^{M_{2}}(y)\right]\right\}}{\operatorname{Tr}\left\{U(\rho \otimes|\xi\rangle\langle\xi|) U^{\dagger}\left[I \otimes E^{M_{1}}(x) E^{M_{2}}(y)\right]\right\}},
$$

provided that the measurement leads to the outcome " $\mathbf{x}=x$ and $\mathbf{y}=y "$.

In Ref. [24], it was proven that for any joint $\operatorname{POVM~} \Pi(x, y)$ there is at least one measuring process $\left(\mathcal{K}, \xi, U, M_{1}, M_{2}\right)$ for $\Pi(x, y)$, which satisfies Eq. (11) for any $x, y \in \mathbf{R}$. By defining $C=U^{\dagger}\left(I \otimes M_{1}\right) U$ and $D=U^{\dagger}\left(I \otimes M_{2}\right) U$ in Eq. (11), we conclude that for any joint $\operatorname{POVM} \Pi(x, y)$ there exist a Hilbert space $\mathcal{K}$, a state vector $\xi$ in $\mathcal{K}$, and commuting observables $C, D$ on $\mathcal{H} \otimes \mathcal{K}$ such that

$$
\Pi(x, y)=\left\langle\xi\left|E^{C}(x) E^{D}(y)\right| \xi\right\rangle
$$

for any $x, y \in \mathbf{R}$. We generally call any quadruple $(\mathcal{K}, \xi, C, D)$ satisfying Eq. (13) for any $x, y \in \mathbf{R}$ the ancilla for the joint $\operatorname{POVM} \Pi(x, y)$.

From Eq. (13), the joint measurement of $A$ and $B$ in state $\psi$ described by $\Pi(x, y)$ is statistically equivalent to the simultaneous measurement of commuting observables $C$ and $D$ in the state $\psi \otimes \xi$. Thus, the ancilla $(\mathcal{K}, \xi, C, D)$ defines the noise $N_{A}$ in the $A$ measurement by the relation

$$
C=A \otimes I+N_{A}
$$

Thus, the noise is the difference between the observable to be measured and the observable actually measured. Since the ancilla is always prepared in the state $\xi$, the partial mean of the noise over the ancilla defines the mean noise operator $n_{A}$ for the $A$ measurement by

$$
n_{A}=\left\langle\xi\left|N_{A}\right| \xi\right\rangle
$$

The rms noise $\epsilon(A)$ for the $A$ measurement in the system state $\psi$ is naturally defined by

$$
\epsilon(A)=\left\langle N_{A}^{2}\right\rangle^{1 / 2},
$$


where $\langle\cdots\rangle$ stands for the mean values in the state $\psi \otimes \xi$. By an easy computations, we have

$$
\begin{aligned}
n_{A} & =O\left(\Pi^{A}\right)-A, \\
\epsilon(A)^{2} & =\left\langle\psi\left|O^{(2)}\left(\Pi^{A}\right)-O\left(\Pi^{A}\right)^{2}+n_{A}^{2}\right| \psi\right\rangle, \\
\left(\Delta N_{A}\right)^{2} & =\epsilon(A)^{2}-\left\langle\psi\left|n_{A}\right| \psi\right\rangle^{2},
\end{aligned}
$$

where $O\left(\Pi^{A}\right)$ and $O^{(2)}\left(\Pi^{A}\right)$ are the first and the second moment operators defined by $O\left(\Pi^{A}\right)=\sum_{x} x \Pi^{A}(x)$ and $O^{2}\left(\Pi^{A}\right)=\sum_{x} x^{2} \Pi^{A}(x)$, and $\Delta N_{A}$ is the standard deviation of the noise $N_{A}$. We also have the following expressions.

$$
\begin{aligned}
n_{A} & =\sum_{x} \Pi^{A}(x)(x-A), \\
\epsilon(A)^{2} & =\sum_{x}\left\|\Pi^{A}(x)^{1 / 2}(x-A) \psi\right\|^{2} .
\end{aligned}
$$

The above relations show that the mean noise operator, the rms noise, and the standard deviation of noise are intrinsic properties of the POVM independent of particular description of the ancilla system.

It can be shown that the POVM $\Pi^{A}$ precisely measures an observable $A$, i.e, $\Pi^{A}(x)=E^{A}(x)$ for any $x$ if and only if $\epsilon(A)=0$ for any state $\psi$ of $A$. The corresponding formulations for the $B$ measurement can be given analogously.

\section{UNIVERSALLY VALID UNCERTAINTY RELATIONS}

Under the above formulation for joint measurements and their noises, the generalized uncertainty relation is obtained as follows (see Refs. [16, 26, 33] for a parallel argument for noise-disturbance uncertainty relations). Since $[C, D]=0$, we obtain $\left[\tilde{A}+N_{A}, \tilde{B}+N_{B}\right]=0$, where $\tilde{A}=A \otimes I$ and $\tilde{B}=B \otimes I$. Then, we have [4]

$$
\left[N_{A}, N_{B}\right]+\left[N_{A}, \tilde{B}\right]+\left[\tilde{A}, N_{B}\right]=-[A, B] \otimes I .
$$

Taking the moduli of means of the both sides and applying the triangular inequality, we have

$$
\left|\left\langle\left[N_{A}, N_{B}\right]\right\rangle\right|+\left|\left\langle\left[\tilde{A}, N_{B}\right]\right\rangle\right|+\left|\left\langle\left[N_{A}, \tilde{B}\right]\right\rangle\right| \geq|\langle\psi|[A, B]| \psi\rangle| .
$$

From Robertson's relation we have

$$
\epsilon(A) \epsilon(B) \geq \Delta N_{A} \Delta N_{B} \geq \frac{1}{2}\left|\left\langle\left[N_{A}, N_{B}\right]\right\rangle\right|
$$

By the relations

$$
\left\langle N_{A} \tilde{B}\right\rangle=\left\langle\psi\left|\left\langle\xi\left|N_{A}\right| \xi\right\rangle\right| B \psi\right\rangle=\left\langle\psi\left|n_{A} B\right| \psi\right\rangle
$$

we have

$$
\left\langle\left[N_{A}, \tilde{B}\right]\right\rangle=\left\langle\psi\left|\left[n_{A}, B\right]\right| \psi\right\rangle
$$

Similarly, we also have

$$
\left\langle\left[\tilde{A}, N_{B}\right]\right\rangle=\left\langle\psi\left|\left[A, n_{B}\right]\right| \psi\right\rangle \text {. }
$$

Therefore, by substituting Eqs. (22), (24), and (25) in Eq. (21), we obtain

$$
\Delta N_{A} \Delta N_{B}+\frac{1}{2}\left|\left\langle\psi\left|\left[n_{A}, B\right]\right| \psi\right\rangle\right|+\frac{1}{2}\left|\left\langle\psi\left|\left[A, n_{B}\right]\right| \psi\right\rangle\right| \geq \frac{1}{2}|\langle\psi|[A, B]| \psi\rangle|,
$$

and the universally valid uncertainty relation for joint measurement

$$
\epsilon(A) \epsilon(B)+\frac{1}{2}\left|\left\langle\psi\left|\left[n_{A}, B\right]\right| \psi\right\rangle\right|+\frac{1}{2}\left|\left\langle\psi\left|\left[A, n_{B}\right]\right| \psi\right\rangle\right| \geq \frac{1}{2}|\langle\psi|[A, B]| \psi\rangle| .
$$

In order to obtain the trade-off among the rms noises $\epsilon(A)$ and $\epsilon(B)$, and the pre-measurement uncertainties $\Delta A$ and $\Delta B$, we apply Robertson's relation to all terms in the left-hand-side of Eq. (21). Then we obtain the generalized uncertainty relation for joint measurements

$$
\epsilon(A) \epsilon(B)+\epsilon(A) \Delta B+\Delta A \epsilon(B) \geq \frac{1}{2}|\langle\psi|[A, B]| \psi\rangle|
$$


From the above, if $\Pi(x, y)$ precisely measures $A$, we have

$$
\Delta A \epsilon(B) \geq \frac{1}{2}|\langle\psi|[A, B]| \psi\rangle| .
$$

Now, we shall discuss how the above relations generalize Heisenberg's relation and related relations obtained previously [3, 4, 6, 7]. We say that the joint $\operatorname{POVM} \Pi(x, y)$ has statistically independent noise for $A$, if the mean noise $\left\langle N_{A}\right\rangle$ does not depend on the input state $\psi$, or equivalently, if the mean noise operator $n_{A}$ is a constant operator, i.e., $n_{A}=r I$ for some $r \in \mathbf{R}$; in this case, we have $n_{A}=\left\langle N_{A}\right\rangle I$. Since the mean noise operator $n\left(N_{A}\right)$ can be interpreted as the conditional expectation of $N_{A}$ conditional upon the state of the object system, the above definition is consistent with a criterion of the statistical independence requiring that the conditional expectation be constant. In fact, if $\Pi(x, y)$ has statistically independent noise for $A$, any observables $X$ of the object and $N_{A}$ are statistically independent in the sense that

$$
\left\langle\tilde{X} N_{A}\right\rangle=\left\langle N_{A} \tilde{X}\right\rangle=\langle\psi|X| \psi\rangle\left\langle N_{A}\right\rangle
$$

for any state $\psi$ of the object; the above relations follow from $\left\langle\tilde{X} N_{A}\right\rangle=\left\langle X \psi\left|\left\langle\xi\left|N_{A}\right| \xi\right\rangle\right| \psi\right\rangle=\left\langle X \psi\left|n_{A}\right| \psi\right\rangle=\langle\psi|X| \psi\rangle\left\langle N_{A}\right\rangle$ and $\left\langle N_{A} \tilde{X}\right\rangle=\left\langle\tilde{X} N_{A}\right\rangle^{*}=\langle\psi|X| \psi\rangle\left\langle N_{A}\right\rangle$.

We say that the joint POVM $\Pi(x, y)$ makes an unbiased measurement of $A$, if the mean output $\sum_{x} x \operatorname{Pr}\{\mathbf{x}=x \| \psi\}$ is equal to the mean $\langle\psi|A| \psi\rangle$ of the observable $A$ in any input state. The above condition is equivalent to the relation $\sum_{x} x \Pi^{A}(x)=A$. From Eq. (17), this is the case if and only if the mean noise operator vanishes, i.e., $n_{A}=0$, so that if $\Pi(x, y)$ makes an unbiased measurement of $A$, then $\Pi(x, y)$ has statistically independent noise for $A$. For the $B$ measurement, the corresponding definitions on statistical independent noise and unbiased measurements are introduced analogously.

Since the relations $n_{A}=r I$ and $n_{B}=r^{\prime} I$ obviously imply $\left[n_{A}, B\right]=\left[A, n_{B}\right]=0$, and hence by Eq. (27) we conclude the following: If the joint POVM $\Pi(x, y)$ has statistically independent noises for both $A$ and $B$, then we have

$$
\epsilon(A) \epsilon(B) \geq \Delta N_{A} \Delta N_{B} \geq \frac{1}{2}|\langle\psi|[A, B]| \psi\rangle|
$$

for any state $\psi$, so that $\Pi(x, y)$ satisfies Heisenberg's relation.

The above relations were previously proven for the unbiased case in Refs. [6, [7].

The standard deviation $\Delta \mathbf{x}$ of the output $\mathbf{x}$ in the state $\psi$ is given by

$$
\Delta \mathbf{x}=\left(\left\langle\psi\left|O^{(2)}\left(\Pi^{A}\right)\right| \psi\right\rangle-\left\langle\psi\left|O\left(\Pi^{A}\right)\right| \psi\right\rangle^{2}\right)^{1 / 2} .
$$

Then, for the ancilla $(\mathcal{K}, \xi, C, D)$ we have $\Delta \mathbf{x}=\Delta C=\Delta\left[\tilde{A}+N_{A}\right]$. Thus, if $\Pi(x, y)$ has statistically independent noise for both $A$ and $B$, we have

$$
\begin{aligned}
& (\Delta \mathbf{x})^{2}=(\Delta A)^{2}+\left(\Delta N_{A}\right)^{2} \geq 2 \Delta A \Delta N_{A}, \\
& (\Delta \mathbf{y})^{2}=(\Delta B)^{2}+\left(\Delta N_{B}\right)^{2} \geq 2 \Delta B \Delta N_{B},
\end{aligned}
$$

and hence apply Eq. (1) and Eq. (31) to the product of the above two inequalities, we have

$$
\Delta \mathbf{x} \Delta \mathbf{y} \geq|\langle\psi|[A, B]| \psi\rangle| .
$$

The above relation has been previously proven for the unbiased case in Ref. [5]. Relation (35) shows that the uncertainties of the outputs increase by the additive fluctuations from the independent noises, and so that the lower bound for the uncertainty product of the outputs is twice as much as that for the measured observables before the measurement. However, the relation $[C, D]=0$ suggests that the dependent noise can reduce the produce $\Delta \mathbf{x} \Delta \mathbf{y}$ arbitrarily small.

\section{CONCLUDING REMARKS}

In this Letter, we have shown that every joint measurement satisfies the relations

$$
\begin{aligned}
\epsilon(A) \epsilon(B)+\epsilon(A) \Delta B+\Delta A \epsilon(B) & \geq \epsilon(A) \epsilon(B)+\frac{1}{2}\left|\left\langle\psi\left|\left[n_{A}, B\right]\right| \psi\right\rangle\right|+\frac{1}{2}\left|\left\langle\psi\left|\left[A, n_{B}\right]\right| \psi\right\rangle\right| \\
& \geq \Delta N_{A} \Delta N_{B}+\frac{1}{2}\left|\left\langle\psi\left|\left[n_{A}, B\right]\right| \psi\right\rangle\right|+\frac{1}{2}\left|\left\langle\psi\left|\left[A, n_{B}\right]\right| \psi\right\rangle\right| \\
& \geq \frac{1}{2}|\langle\psi|[A, B]| \psi\rangle| .
\end{aligned}
$$


If the apparatus has statistically independent noises for both $A$ and $B$, then we have $\left\langle\psi\left|\left[n_{A}, B\right]\right| \psi\right\rangle=\left\langle\psi\left|\left[A, n_{B}\right]\right| \psi\right\rangle=0$, so that the above relations are reduced to Heisenberg's relation

$$
\epsilon(A) \epsilon(B) \geq \Delta N_{A} \Delta N_{B} \geq \frac{1}{2}|\langle\psi|[A, B]| \psi\rangle| .
$$

In this case, the product of the output standard deviations has the twice as much lower bound as the input standard deviations, i.e.,

$$
\Delta \mathbf{x} \Delta \mathbf{y} \geq|\langle\psi|[A, B]| \psi\rangle| .
$$

In Heisenberg's relation a precise measurement of one observable excludes the measurement of the other noncommuting observable even with any finite noise. However, from $\epsilon(A)=0$ our relation concludes

$$
\Delta A \epsilon(B) \geq \frac{1}{2}|\langle\psi|[A, B]| \psi\rangle|,
$$

which suggests the possibility that we can overcome the Heisenberg's limitation of the measurement when one of the noises are not statistically independent.

In the preceding discussions, we assume that $\mathcal{H}$ is finite dimensional and $\mathbf{x}$ and $\mathbf{y}$ take only finite number of values. However, the generalization to infinite dimensional case allowing continuous valued $\mathbf{x}, \mathbf{y}$ is rather straightforward. In the general case, the POVM is defined by any family $\{\Pi(x, y) \mid x, y \in \mathbf{R})\}$ of positive operators on $\mathcal{H}$ satisfying (i) $0 \leq \Pi(x, y) \leq \Pi\left(x^{\prime}, y^{\prime}\right) \leq I$ if $x \leq x^{\prime}$ and $y \leq y^{\prime}$, (ii) $\lim _{x \rightarrow-\infty, y \rightarrow-\infty} \Pi(x, y)=0$, (iii) $\lim _{x \rightarrow \infty, y \rightarrow \infty} \Pi(x, y)=I$, and (iv) $\lim _{x \rightarrow a+0, y \rightarrow b+0} \Pi(x, y)=\Pi(a, b)$ for any $a, b \in \mathbf{R}$. Then, the joint probability of obtaining the outcomes $\mathbf{x} \leq x$ and $\mathbf{y} \leq y$ in the state $\psi$ is described by $\operatorname{Pr}\{\mathbf{x} \leq x, \mathbf{y} \leq y \| \psi\}=\langle\psi|\Pi(x, y)| \psi\rangle$. Then, the marginal POVMs, and moment operators are defined by $\Pi^{A}(x)=\lim _{y \rightarrow \infty} \Pi(x, y), O\left(\Pi^{A}\right)=\int_{\mathbf{R}} x d \Pi^{A}(x), O\left(\Pi^{A}\right)=\int_{\mathbf{R}} x^{2} d \Pi^{A}(x)$, and so on; see Ref. [6] for the detail. Then, we have the same formulas as Eqs. (36)-(41), as long as all the relevant terms are finite.

In Ref. [16], it has been shown that the noise for an observable $A$ and the disturbance for another observable $B$ in a single output measurement using an apparatus $\mathbf{A}(\mathbf{x})$ can be identified with the two noises of $A$ and $B$ in the joint measurement of $A$ and $B$ using apparatus $\mathbf{A}(\mathbf{x}, \mathbf{y})$ obtained by the successive measurement consisting of the measurement using $\mathbf{A}(\mathbf{x})$ immediately followed by a precise $B$ measurement using an apparatus $\mathbf{A}(\mathbf{y})$. In this way, the noise and disturbance relations previously obtained in Ref. [16] have been generalized in the model-independent formulation to the uncertainty relations for joint measurements in the present Letter.

Now we return to the joint measurement of $P+P^{\prime}$ and $Q$ for two independent pairs $(Q, P)$ and $\left(Q^{\prime}, P^{\prime}\right)$ of conjugate observables. Let $\psi=\psi_{1} \otimes \psi_{2}$. Since $\left[P+P^{\prime}, Q\right]=i \hbar$, from Eq. [28], we generally have

$$
\epsilon\left(P+P^{\prime}\right) \epsilon(Q)+\epsilon\left(P+P^{\prime}\right) \Delta Q+\Delta\left(P+P^{\prime}\right) \epsilon(Q) \geq \frac{\hbar}{2} .
$$

The above relation does not exclude the possibility of having $\epsilon\left(P+P^{\prime}\right)=0$, and in this case we have

$$
\Delta\left(P+P^{\prime}\right) \epsilon(Q) \geq \frac{\hbar}{2} .
$$

Let $\psi=\psi_{1} \otimes \psi_{2}$. Then, we have $\left[\Delta\left(P+P^{\prime}\right)\right]^{2}=(\Delta P)^{2}+\left(\Delta P^{\prime}\right)^{2}$. Thus we have

$$
\epsilon(Q)^{2} \geq \frac{\hbar^{2}}{4(\Delta P)^{2}+4\left(\Delta P^{\prime}\right)^{2}}
$$

The above relation gives general limit for the measurement of position $Q$ without disturbing the total momentum $P+P^{\prime}$. For the example in the beginning of the present Letter, we have $\epsilon(Q)=\Delta Q^{\prime}$, and this would be optimal if $P$ could be prepared definitely, i.e., $\Delta P=0$, and $Q$ is prepared in the minimum uncertainty state, i.e., $\Delta Q^{\prime} \Delta P^{\prime}=$ $\hbar / 2$. Therefore, the present results on generalizing Heisenberg's relation settles the confusion for the accuracy of measurement in the presence of a conserved quantity; see [31, 32] for relevant discussions.

\section{Acknowledgements}

This work was supported by the Strategic Information and Communications R\&D Promotion Scheme of the MPHPT of Japan, by the CREST project of the JST, and by the Grant-in-Aid for Scientific Research of the JSPS.

[1] W. Heisenberg, Z. Phys. 43, 172 (1927). 
[2] W. Heisenberg, The Physical Principles of the Quantum Theory (University of Chicago Press, Chicago, 1930) [Reprinted by Dover, New York $(1949,1967)]$.

[3] E. Arthurs and J. L. Kelly, Jr., Bell. Syst. Tech. J. 44, 725 (1965).

[4] Y. Yamamoto and H. A. Haus, Rev. Mod. Phys. 58, 1001 (1986).

[5] E. Arthurs and M. S. Goodman, Phys. Rev. Lett. 60, 2447 (1988).

[6] M. Ozawa, Quantum limits of measurements and uncertainty principle, in Quantum Aspects of Optical Communications, edited by C. Bendjaballah et al., Lecture Notes in Physics 378, pages 3-17 (Springer, Berlin, 1991).

[7] S. Ishikawa, Rep. Math. Phys. 29, 257 (1991).

[8] C. Y. She and H. Heffner, Phys. Rev. 152, 1103 (1966).

[9] H. P. Yuen, Phys. Lett. A 91, 101 (1982).

[10] H. P. Yuen, Phys. Rev. Lett. 51, 719 (1983).

[11] H. Martens and W. M. de Muynck, Found. Phys. 20, 357 (1990).

[12] M. Ozawa, Phys. Rev. Lett. 60, 385 (1988).

[13] M. Ozawa, Realization of measurement and the standard quantum limit, in Squeezed and Nonclassical Light, edited by P. Tombesi and E. R. Pike, pages 263-286 (New York, Plenum, 1989).

[14] M. Ozawa, Phys. Lett. A 282, 336 (2001).

[15] M. Ozawa, Phys. Lett. A 299, 1 (2002).

[16] M. Ozawa, Phys. Rev. A 67, 042105 (2003).

[17] E. H. Kennard, Z. Phys. 44, 326 (1927).

[18] H. P. Robertson, Phys. Rev. 34, 163 (1929).

[19] L. E. Ballentine, Rev. Mod. Phys. 42, 358 (1970).

[20] A. Peres, Quantum Theory: Concepts and Methods (Kluwer Academic, Dordrecht, 1993).

[21] C. W. Helstrom, Quantum Detection and Estimation Theory (Academic, New York, 1976).

[22] E. B. Davies, Quantum Theory of Open Systems (Academic, London, 1976).

[23] A. S. Holevo, Probabilistic and Statistical Aspects of Quantum Theory (North-Holland, Amsterdam, 1982).

[24] M. Ozawa, J. Math. Phys. 25, 79 (1984).

[25] M. A. Nielsen and I . L. Chuang, Quantum Computation and Quantum Information (Cambridge University Press, Cambridge, 2000).

[26] M. Ozawa, Uncertainty relations for noise and disturbance in generalized quantum measurements, online preprint: LANL quant-ph/0307057 (2003).

[27] E. P. Wigner, Z. Phys. 133, 101 (1952).

[28] H. Araki and M. M. Yanase, Phys. Rev. 120, 622 (1960).

[29] M. M. Yanase, Phys. Rev. 123, 666 (1961).

[30] E. P. Wigner, Am. J. Phys. 31, 6 (1963).

[31] M. Ozawa, Phys. Rev. Lett. 67, 1956 (1991).

[32] M. Ozawa, Phys. Rev. Lett. 88, 050402 (2002).

[33] M. Ozawa, Phys. Lett. A 318 (2003), 21. 